\title{
Localization in non-Hermitian asymmetric rhombic lattice
}

\author{
S. M. Zhang $\odot$ and L. Jin $\odot^{*}$ \\ School of Physics, Nankai University, Tianjin 300071, China
}

(Received 2 May 2020; revised 22 June 2020; accepted 6 July 2020; published 23 July 2020)

\begin{abstract}
We propose a non-Hermitian rhombic lattice and investigate the Aharonov-Bohm caging, which forms at the exceptional point (EP) of the rhombic lattice at which all the lattice eigenstates are coalesced. The compact localized eigenstates and the localization dynamics are investigated. The excitation intensity can be constant or can polynomially increase with time as a consequence of the peculiar dynamics at the EP. The excitation can be completely localized in the two diamond plaquettes of the rhombic lattices and the localization area can be controlled by the gain and loss. Our findings shed light on the Aharonov-Bohm cage in the non-Hermitian physics and are beneficial for light engineering in the non-Hermitian metamaterials.
\end{abstract}

DOI: 10.1103/PhysRevResearch.2.033127

\section{INTRODUCTION}

Flat bands, which are dispersionless and independent of momentum, have displayed many exotic properties during the past few decades [1-16]. The systems with a flat band are fabricated in various physical systems [11]. The diamond chain, with a zero energy flat band and two dispersive bands, possesses an energy-dependent localization length scaling [3]. Also, the one-dimensional (1D) tight-binding lattices hold an anomalous minimum of localization length near an isolated flat band even in the presence of disorders [17]. The particle interaction in the diamond chain can induce delocalization [18]; in a Hubbard model of the diamond chain, many interesting properties including flat-band ferromagnetism, correlated half metal, and insulator-metal transition are displayed [19].

The development of Aharonov-Bohm (AB) cages in photonic systems promotes light control, for example, in the lattices with a quasi-1D rhombic geometry. Different from the dynamic localization in ordered systems, an unsalted mechanism requiring synthetic gauge fields results in the destructive interference in the photonic lattices through modulating the waveguides or resonators [20]. In particular, the localization dynamics of $\mathrm{AB}$ caging is observed in the experimentally realized photonic rhombic lattice with a uniform flux $\pi$ in each plaquette [21]. Also, theoretical and experimental illustration on an $\mathrm{AB}$ cage chain helps to explore the topological properties of topological insulators [22]. The nonlinear effects are investigated in the quasi-1D rhombic lattice $[23,24]$ and the two-dimensional (2D) dice lattice [25].

The $\mathcal{P} \mathcal{T}$-symmetric systems are invariant under the combined spatial reflection $(\mathcal{P})$ and time-reversal $(\mathcal{T})$ operations,

\footnotetext{
*jinliang@ nankai.edu.cn

Published by the American Physical Society under the terms of the Creative Commons Attribution 4.0 International license. Further distribution of this work must maintain attribution to the author(s) and the published article's title, journal citation, and DOI.
}

but not necessarily having the individual $\mathcal{P}$ and $\mathcal{T}$ symmetries at the same time. The non-Hermitian systems may possess entirely real spectra, determined by the $\mathcal{P} \mathcal{T}$ symmetry of their eigenstates. The $\mathcal{P} \mathcal{T}$ symmetry breaks at the nonHermitian phase transition point, which is the exceptional point (EP) that uniquely exists in non-Hermitian systems and has many intriguing features and potential applications including unidirectional lasing, optical sensing, and robust topological energy transfer [26-32]. With the rapid growth of non-Hermitian physics, researchers have paid attention to the $\mathcal{P} \mathcal{T}$-symmetric systems with flat bands [33,34]. The bipartite sublattice symmetry leads to the $\mathcal{P} \mathcal{T}$-symmetric flat bands in the non-Hermitian environments, and the corresponding compact localized states (CLSs) are nondiffracting and robust to disorders [35]. Disorders with $\mathcal{P} \mathcal{T}$ symmetry enhance the degeneracy of flat bands in a 1D Lieb lattice, which strongly influence the dispersive bands rather than the flat bands [34]. Under the non-Hermitian particle-hole symmetry with tunable gain and loss, flat bands in such a system are composed of photonic zero modes [36]. A flat band is formed when changing the degree of non-Hermiticity in the triangular $\mathcal{P} \mathcal{T}$-symmetric lattices, and the light localization is controllable [37]. In the non-Hermitian systems, the CLSs excited at any position are observable with the existence of flat bands in a tectonic optical system under $\mathcal{P} \mathcal{T}$ symmetry [38]. Without breaking the destructive interference, the gain and loss can be introduced to construct the $\mathcal{P} \mathcal{T}$-symmetric flat bands in both the $1 \mathrm{D}$ and $2 \mathrm{D}$ systems $[39,40]$.

In this paper, we investigate the asymmetric rhombic lattice, which has imbalanced arms in the diamond plaquette. The asymmetric rhombic lattice possesses a spectrum fully consisting of flat bands under the destructive interference when each diamond plaquette encloses a half magnetic flux quantum. The compactly localized eigenstates in the asymmetric rhombic lattice are analytically studied. The localization effect and Aharonov-Bohm caging are discussed. A non-Hermitian rhombic lattice is proposed based on the localization in the asymmetric rhombic lattice. The proposed non-Hermitian rhombic lattice also supports an entirely real spectrum as well as the Aharonov-Bohm caging; however, the 
(a)

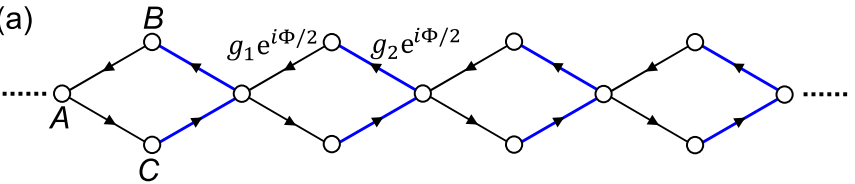

(b)

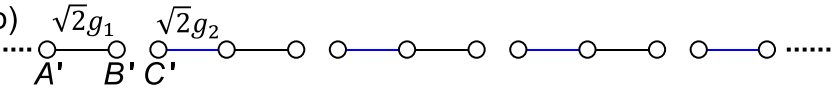

(c)

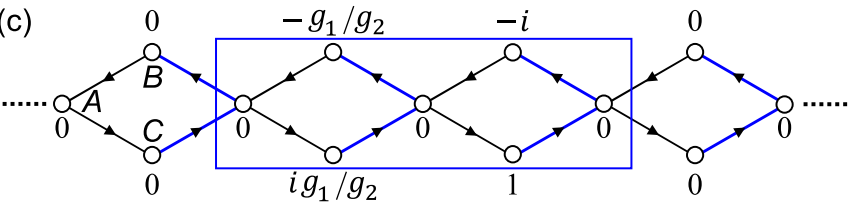

(d)

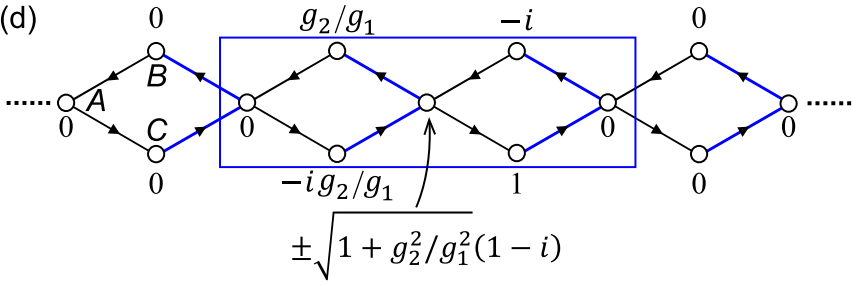

FIG. 1. (a) Schematic of the asymmetric rhombic lattice enclosed $\pi$ magnetic flux in each plaquette, $\Phi=\pi / 2$. (b) Schematic of the equivalent lattice possessing identical spectrum with (a). Compact localized states of flat bands with (c) zero energy and (d) nonzero energy. In (d) the wave-function amplitude at the center site is $\pm \sqrt{1+g_{2}^{2} / g_{1}^{2}}(1-i)$ for the nonzero energy $\pm \sqrt{2 g_{1}^{2}+2 g_{2}^{2}}$.

CLSs are coalesced and the localization area of the excitation can be finely tuned by the non-Hermitian gain and loss in the proposed non-Hermitian rhombic lattice. The numerical simulations of the Aharonov-Bohm caging dynamics are performed; the excitation intensity can be finite or polynomially increase with time. The time evolved excitation intensity and the localization configuration highly depend on the gain and loss. We systematically investigate the tunable localization dynamics in the non-Hermitian asymmetric rhombic lattice.

The remainder of the paper is organized as follows. In Sec. II, the asymmetric rhombic lattice model is presented. In Sec. III, the eigenstates of the rhombic lattice are investigated. In Secs. IV and V, we depict the non-Hermitian rhombic lattice and the localization dynamics, respectively. In Sec. V, the conclusions are summarized.

\section{ASYMMETRIC RHOMBIC LATTICE}

We depict the schematic of the asymmetric rhombic lattice in Fig. 1(a). The asymmetric rhombic lattice has three sublattices $A, B$, and $C$. The sublattice $A$ couples to sublattices $B$ and $C$ simultaneously; the sublattices $B$ and $C$ only couple to sublattice $A$. The sublattices form the diamond plaquettes aligned in the horizontal direction as a quasi-1D lattice. The coupling strengths are $g_{1}$ and $g_{2}$, where $g_{1}\left(g_{2}\right)$ is the coupling between the site of sublattice $A$ and the sites of sublattices $B$ and $C$ on the right (left) side of the site of sublattice $A$. In the horizontal direction, the couplings $g_{1}$ and $g_{2}$ are staggered. The arrow in the schematic indicates the additional phase factor $e^{i \pi / 4}$ that is accumulated in the tunneling process when photons or particles tunnel along the direction of each arrow belonging to the nonreciprocal coupling $g_{1} e^{ \pm i \pi / 4}$ (black arrow) or $g_{2} e^{ \pm i \pi / 4}$ (blue arrow). In the diamond plaquette, the arrow directions are identical; all of them are in the counterclockwise direction. Thus, each diamond plaquette encloses $\pi$ magnetic flux as a consequence of the Peierls phase factor $e^{i \pi / 4}$ in the front of the four couplings. The photonic lattice provides an ideal platform for investigating the flat band. In the coupled waveguide lattice, the linear gradient in the propagation constants by circularly bending the waveguides and the periodical modulation in the propagation constants at the frequency of the gradient induce the nonreciprocal coupling with Peierls phase factor [20]. The symmetric rhombic lattice (at $g_{1}=g_{2}$ ) threaded by synthetic magnetic flux $\pi$ in each diamond plaquette has been experimentally demonstrated in the coupled waveguide lattice, which is fabricated by the direct femtosecond laser writing technique [21]. Alternatively, the $\pi$ magnetic flux in the diamond plaquette is created by engineering one of the four couplings to be a negative coupling, possessing an opposite sign with respect to the other three couplings. This is assisted by an auxiliary waveguide with fine-tuning refractive index, positioning between two waveguides and inducing an effective negative coupling between them [22]. Both methods can be directly used to realize the asymmetric rhombic waveguide lattice. The coupling strengths depend on the distance of the waveguides; a staggered arrangement of the distances between the waveguides in the rhombic lattice generates staggered coupling $g_{1} \neq g_{2}$. Also, the synthetic magnetic flux is experimentally realized in the coupled resonator lattice [41]. The neighbor primary resonators are directly coupled to the link resonator between them. Photons tunnel between the neighbor primary resonators through the link resonator, which induces an effective coupling between the neighbor primary resonators. The optical path-length difference for photons tunneling in the forward and backward directions induces the nonreciprocal coupling with Peierls phase factor [42]. Thus, the quasi-1D asymmetric rhombic lattice also models the photonic lattice of coupled resonators.

Applying the Fourier transformation, the Hamiltonian in real space is transformed to the momentum space

$$
H_{k}=\left(\begin{array}{ccc}
0 & G_{1}+G_{2}^{*} e^{-i k} & G_{1}^{*}+G_{2} e^{-i k} \\
G_{1}^{*}+G_{2} e^{i k} & 0 & 0 \\
G_{1}+G_{2}^{*} e^{i k} & 0 & 0
\end{array}\right),
$$

where $G_{1}=g_{1} e^{i \pi / 4}$ and $G_{2}=g_{2} e^{i \pi / 4}$. The energy bands of $H_{k}$ are $E_{k}=0, \pm \sqrt{2 g_{1}^{2}+2 g_{2}^{2}}$. The three energy bands are all dispersionless; thus, the asymmetric rhombic lattice has an entirely flat spectrum and supports Aharonov-Bohm caging when the magnetic flux enclosed in the diamond plaquette is $\pi$.

In addition to elucidating the asymmetric rhombic lattice through calculating the spectrum, we introduce an equivalent three-band lattice for the asymmetric rhombic lattice. The schematic of the equivalent three-band lattice is presented in Fig. 1(b), which provides a clear physical picture for the essential features of the asymmetric rhombic lattice. In real space, the equivalent three-band lattice Hamiltonian $H^{\prime}$ is obtained from the asymmetric rhombic lattice Hamiltonian $H$ under a unitary transformation $H^{\prime}=\left(I_{N} \otimes U\right)^{-1} H\left(I_{N} \otimes U\right)$, 
where $I_{N}$ is the $N \times N$ identical matrix and $N$ is the number of the triangular unit cells. The $j$ th unit cell includes three sites $\left\{A_{j}, B_{j}, C_{j}\right\} . U$ is a $3 \times 3$ unitary matrix in the form of

$$
U=\left(\begin{array}{ccc}
1 & 0 & 0 \\
0 & e^{-i \pi / 4} \sqrt{2} / 2 & i e^{-i \pi / 4} \sqrt{2} / 2 \\
0 & i e^{-i \pi / 4} \sqrt{2} / 2 & e^{-i \pi / 4} \sqrt{2} / 2
\end{array}\right) .
$$

In practice, the most intriguing fact is that the unitary transformation $I_{N} \otimes U$ merges the sublattices $B$ and $C$ in the same unit cell and creates decoupled sublattices $B^{\prime}$ and $C^{\prime}$ in a new unit cell. Notably, the equivalent lattice in Fig. 1(b) is constituted by the isolated trimers; the trimers are all identical and the three sites in each trimer are coupled under coupling strengths $\sqrt{2} g_{1}$ and $\sqrt{2} g_{2}$. The trimer has three energies 0 and $\pm \sqrt{2 g_{1}^{2}+2 g_{2}^{2}}$. The spectrum for the equivalent lattice is composed by the energy levels of $N$ isolated trimers for an asymmetric rhombic lattice with $N$ unit cells; thus, each band has $N$ degenerate levels. From the equivalent lattice, we notice that the spectrum is fully constituted by the flat bands and the eigenstates are definitely compactly localized in each isolated trimer; consequently, the corresponding eigenstates of the asymmetric rhombic lattice are compactly localized in the related five sites centered at one site of sublattice $A$ associated with two sites of sublattices $B$ and $C$, respectively, in its two neighbor diamond plaquettes.

\section{COMPACT LOCALIZED EIGENSTATES}

The un-normalized eigenstates of the flat bands are CLSs and they distribute in two neighbor plaquettes. The wavefunction amplitude for each site of the CLSs without normalization is shown in Figs. 1(c) and 1(d). Notably, the asymmetric coupling results in the imbalance between the wave functions of the left plaquette and the right plaquette. Each two adjacent plaquettes support three linear independent CLSs, and these CLSs are orthogonal and form the complete set of eigenstates.

The discrete Schrödinger equations for the asymmetric rhombic lattice are

$$
\begin{aligned}
i \dot{\psi}_{A_{j}}= & g_{1} e^{i \pi / 4} \psi_{B_{j}}+g_{1} e^{-i \pi / 4} \psi_{C_{j}} \\
& +g_{2} e^{-i \pi / 4} \psi_{B_{j-1}}+g_{2} e^{i \pi / 4} \psi_{C_{j-1}}, \\
i \dot{\psi}_{B_{j}}= & g_{1} e^{-i \pi / 4} \psi_{A_{j}}+g_{2} e^{i \pi / 4} \psi_{A_{j+1}}, \\
i \dot{\psi}_{C_{j}}= & g_{1} e^{i \pi / 4} \psi_{A_{j}}+g_{2} e^{-i \pi / 4} \psi_{A_{j+1}},
\end{aligned}
$$

where the wave functions at the $j$ th unit cell of the lattice are $\psi_{A_{j}}, \psi_{B_{j}}$, and $\psi_{C_{j}}$.

The localization of the CLSs can be seen from the viewpoint of interference. The CLSs are distributed and confined in the five-site-cross configurations in the two neighbor plaquettes for all the flat bands, in contrast to the flat-band CLSs that compactly localize in a single unit cell of the other diamond lattices [11,39]. As shown in Figs. 1(c) and 1(d), each of the three types of CLSs centers at the middle site $A$ of two unit cells.

For the zero energy CLS in Fig. 1(c), the wave functions of the left $B$ and left $C$ sites destructively interfere at the left $A$ site, that is,

$$
\begin{aligned}
& \left(g_{1} e^{i \pi / 4}\right) \psi_{B_{j}}+\left(g_{1} e^{-i \pi / 4}\right) \psi_{C_{j}} \\
& \quad=\left(g_{1} e^{i \pi / 4}\right)\left(-g_{1} / g_{2}\right)+\left(g_{1} e^{-i \pi / 4}\right)\left(i g_{1} / g_{2}\right)=0 .
\end{aligned}
$$

The wave functions of the right $B$ and right $C$ sites destructively interfere at the right $A$ site, that is,

$$
\begin{aligned}
& g_{2} e^{-i \pi / 4} \psi_{B_{j-1}}+g_{2} e^{i \pi / 4} \psi_{C_{j-1}} \\
& =\left(g_{2} e^{-i \pi / 4}\right)(-i)+\left(g_{2} e^{i \pi / 4}\right)(1)=0 .
\end{aligned}
$$

Thus, the wave-function amplitudes of the left and the right $A$ sites both vanish, and the CLSs are confined in the fivesite-cross configuration inside two neighbor plaquettes as elucidated in Fig. 1(c). Furthermore, the contributions of the left $B(C)$ site and the right $C(B)$ site in the interference cancel at the central $A$ site, $\left(g_{2} e^{-i \pi / 4}\right)\left(-g_{1} / g_{2}\right)+\left(g_{1} e^{-i \pi / 4}\right)(1)=0$ $\left[\left(g_{2} e^{i \pi / 4}\right)\left(i g_{1} / g_{2}\right)+\left(g_{1} e^{i \pi / 4}\right)(-i)=0\right]$. Thus, the zero energy CLS has destructive interference at all sites of the sublattice $A$. We can check that the zero energy CLS satisfies the steady-state Schrödinger equations:

$$
\begin{aligned}
E \psi_{A_{j}}= & g_{1} e^{i \pi / 4} \psi_{B_{j}}+g_{1} e^{-i \pi / 4} \psi_{C_{j}} \\
& +g_{2} e^{-i \pi / 4} \psi_{B_{j-1}}+g_{2} e^{i \pi / 4} \psi_{C_{j-1}}, \\
E \psi_{B_{j}}= & g_{1} e^{-i \pi / 4} \psi_{A_{j}}+g_{2} e^{i \pi / 4} \psi_{A_{j+1}}, \\
E \psi_{C_{j}}= & g_{1} e^{i \pi / 4} \psi_{A_{j}}+g_{2} e^{-i \pi / 4} \psi_{A_{j+1}} .
\end{aligned}
$$

For the nonzero energy CLS in Fig. 1(d), notice that the ratio between the CLS wave-function amplitudes $B$ and $C$ in the left plaquette is $1:(-i)$, which results in the destructive interference at the left $A$ site; the ratio between the CLS wavefunction amplitudes $B$ and $C$ in the right plaquette is $(-i): 1$, which results in the destructive interference at the right $A$ site.

For the nonzero energy CLS of $E=\sqrt{2 g_{1}^{2}+2 g_{2}^{2}}$, the steady-state Schrödinger equations (8)-(10) for the central five sites of the two plaquettes can be verified as follows. Notably, the wave function of the central $A$ site as marked in Fig. 1(d) can be rewritten as $\left(E / g_{1}\right)(1-i) / \sqrt{2}$. The left $B$ site satisfies

$$
g_{2} e^{i \pi / 4}\left(E / g_{1}\right)(1-i) / \sqrt{2}=E\left(g_{2} / g_{1}\right),
$$

the left $C$ site satisfies

$$
g_{2} e^{-i \pi / 4}\left(E / g_{1}\right)(1-i) / \sqrt{2}=E\left(-i g_{2} / g_{1}\right),
$$

the right $B$ site satisfies

$$
g_{1} e^{-i \pi / 4}\left(E / g_{1}\right)(1-i) / \sqrt{2}=E(-i),
$$

the right $C$ site satisfies

$$
g_{1} e^{i \pi / 4}\left(E / g_{1}\right)(1-i) / \sqrt{2}=E(1),
$$

and the amplitudes of the left $A$ site and the right $A$ site are both zeros; thus, the steady-state Schrödinger equations Eqs. (9) and (10) are verified. The central $A$ site satisfies

$$
\begin{aligned}
g_{1} e^{i \pi / 4}(-i)+g_{1} e^{-i \pi / 4}(1) & \\
& +g_{2} e^{-i \pi / 4}\left(g_{2} / g_{1}\right)+g_{2} e^{i \pi / 4}\left(-i g_{2} / g_{1}\right) \\
= & (1-i) \sqrt{2} g_{1}\left(1+g_{2}^{2} / g_{1}^{2}\right),
\end{aligned}
$$


thus the steady-state Schrödinger equation (8) is verified. Similarly, it is straightforward to verify that the nonzero energy CLS with $E=-\sqrt{2 g_{1}^{2}+2 g_{2}^{2}}$ as marked in Fig. 1(d) is also the steady-state solution using the same approach. Therefore, both the zero and nonzero flat bands satisfy the steady-state Schrödinger equations of the asymmetric rhombic lattice.

Notably, every two neighbor plaquettes support three CLSs with different energies. The asymmetric rhombic lattice with $N$ number of unit cells has $N$ pairs of two neighbor plaquettes. Thus, the total number of CLSs presented in Figs. 1(c) and $1(\mathrm{~d})$ is $3 N$. The total $3 N$ CLSs are linearly independent configurations centered at the sites of sublattice $A$; they are the eigenstates of the flat bands, being orthogonal to each other, and constitute a complete set of eigenstates of the rhombic lattice.

In the Hermitian lattice, the CLSs with different energies are apparently orthogonal, which can be directly verified from the eigenstates shown in Figs. 1(c) and 1(d). The degenerate CLSs are also orthogonal. This can be verified from the fact that two degenerate CLSs may share at most two sites when considering two CLSs that are closely localized, that is, a site $B$ and a site $C$ in the same plaquette; even in this situation, the two degenerate states are orthogonal, that is, $\left(-g_{1} / g_{2}\right)(-i)^{*}+\left(i g_{1} / g_{2}\right)(1)^{*}=0$ for the zero energy flat band and $\left(g_{2} / g_{1}\right)(-i)^{*}+\left(-i g_{2} / g_{1}\right)(1)^{*}=0$ for the nonzero energy flat band.

Any initial state is a superposition of the CLSs in the configurations of Figs. 1(c) and 1(d). Thus, any initial state will be completely confined in these five-site-cross configurations.

\section{NON-HERMITIAN ASYMMETRIC RHOMBIC LATTICE}

Based on the isolated trimers shown in Fig. 1(b), we propose non-Hermitian isolation through considering additional unidirectional coupling between the neighbor isolated trimers as shown in Fig. 2(a). Photons or particles only one-way tunnel between the sites with the unidirectional coupling along the direction indicated by the pink arrows; in the opposite direction, the photon or particle tunneling is prevented. In this manner, the trimer lattice in Fig. 2(a) is a 1D non-Hermitian lattice at the exceptional point because of the Jordan block form of unidirectional couplings.

In contrast to the Hermitian isolated trimers, the isolation occurs only within the areas that are connected by two oppositely oriented unidirectional couplings toward the inside. In Fig. 2(a), the unidirectional couplings alternatively present between the trimers. Two nearest-neighbor trimer cells either have two inward unidirectional couplings or have two outward unidirectional couplings. Therefore, the confinement occurs for every other trimer cell with two inward unidirectional couplings; then, the tunneling only occurs from the outside to the inside and the flow is inward; consequently, the initial excitations in such type of trimers will be completely confined. Alternatively, the initial excitations in the trimer with outward unidirectional couplings will tunnel to its neighbor trimers and be confined in their neighbor trimer cells.

We further elucidate the non-Hermitian isolation to proceed with our discussion. We introduce the quasi-1D $\mathcal{P} \mathcal{T}$-symmetric non-Hermitian asymmetric rhombic lattice, schematically illustrated in Fig. 2(b), which is equivalent to
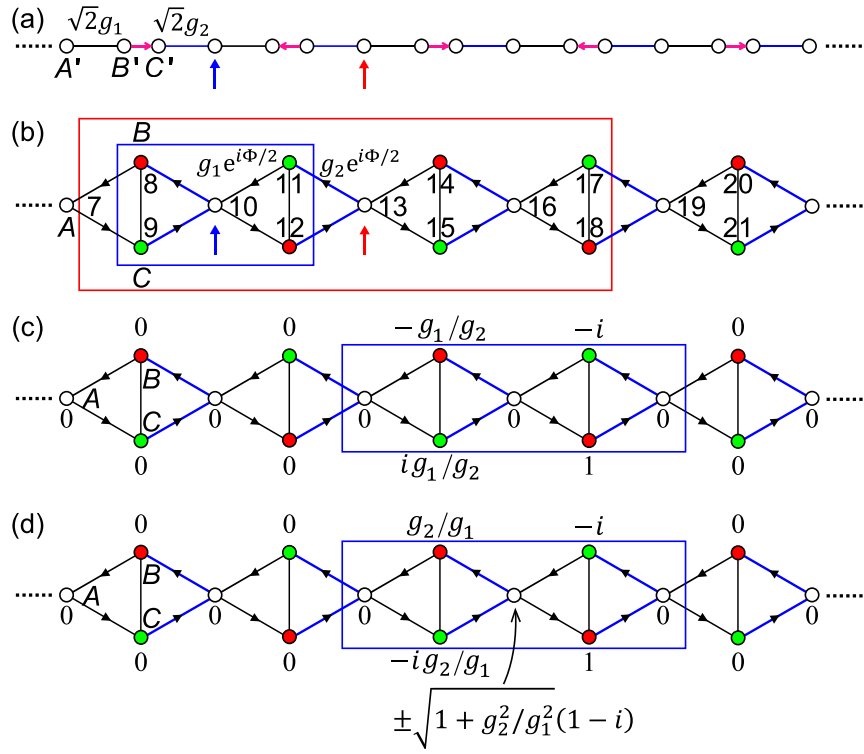

FIG. 2. (a) Schematic of the non-Hermitian isolation; the unidirectional coupling strength is $2 \gamma$. (b) Schematic of the equivalent $\mathcal{P} \mathcal{T}$-symmetric non-Hermitian asymmetric rhombic lattice that possesses identical spectrum with (a), $\Phi=\pi / 2$. The site in red (green) has the gain (loss) with rate $\gamma$; the coupling in between is $\delta=\gamma$. (c) Zero energy CLS. (d) Nonzero energy CLS. In (d), the wave-function amplitude at the center site is $\pm \sqrt{1+g_{2}^{2} / g_{1}^{2}}(1-i)$ for the nonzero energy $\pm \sqrt{2 g_{1}^{2}+2 g_{2}^{2}}$.

the 1D non-Hermitian isolated trimer lattice with unidirectional couplings that is schematically illustrated in Fig. 2(a). The quasi-1D $\mathcal{P} \mathcal{T}$-symmetric non-Hermitian asymmetric rhombic lattice is mathematically obtained through applying the inverse transformation of $\left(I_{N} \otimes U\right)$ to the non-Hermitian isolated trimer lattice Hamiltonian. Therefore, the physics of the two Hamiltonians are identical.

The Hamiltonian of the quasi-1D non-Hermitian asymmetric rhombic lattice in real space [Fig. 2(b)] reads

$$
\begin{aligned}
\mathcal{H}_{\mathrm{RL}}= & \sum_{j}\left(G_{1} a_{2 j-1}^{\dagger} b_{2 j-1}+G_{1}^{*} a_{2 j-1}^{\dagger} c_{2 j-1}+G_{1} a_{2 j}^{\dagger} b_{2 j}\right. \\
& +G_{1}^{*} a_{2 j}^{\dagger} c_{2 j}+G_{2}^{*} a_{2 j}^{\dagger} b_{2 j-1}+G_{2} a_{2 j}^{\dagger} c_{2 j-1} \\
& +G_{2}^{*} a_{2 j-1}^{\dagger} b_{2 j-2}+G_{2} a_{2 j-1}^{\dagger} c_{2 j-2} \\
& \left.+\delta b_{2 j-1}^{\dagger} c_{2 j-1}+\delta b_{2 j}^{\dagger} c_{2 j}+\text { H.c. }\right) \\
& +i \gamma b_{2 j-1}^{\dagger} b_{2 j-1}-i \gamma c_{2 j-1}^{\dagger} c_{2 j-1} \\
& -i \gamma b_{2 j}^{\dagger} b_{2 j}+i \gamma c_{2 j}^{\dagger} c_{2 j},
\end{aligned}
$$

where $a^{\dagger}, b^{\dagger}$, and $c^{\dagger}(a, b$, and $c)$ are the creation (annihilation) operators for the sublattice $A, B$, and $C$. The subscript is the site number of the sublattice.

In the schematic of Fig. 2(b), the sites in red and green indicate that additional gain and loss $\pm i \gamma$ are present in the sublattices $B$ and $C$. The black line represents the additional coupling $\delta$ between the two sublattices $B$ and $C$. The gain and loss rates equal to the coupling $\gamma=\delta$, according to the unidirectional coupling of the 1D Hamiltonian. It is worth emphasizing that the gain and loss distribution in the sublattices 
$B$ and $C$ depends on the direction of the unidirectional coupling, or, alternatively, the distribution of gain and loss determines the direction of the equivalent unidirectional coupling in the equivalent 1D lattice. The quasi-1D non-Hermitian asymmetric lattice is readily experimentally attainable in the coupled waveguide lattice. The Hermitian symmetric rhombic lattice threaded by synthetic magnetic flux has been realized $[21,22]$. The synthetic magnetic flux is effectively created through tuning the relative phase of the periodic modulation on the off-diagonal frequencies of the coupled waveguides as previously discussed. In the waveguides, the gain can be introduced through extra pumping [43-45]. The loss can be intentionally introduced through adding extra absorption such as fabricating radiative losses, cutting the waveguides, or sticking absorbing materials [46-49]. The non-Hermitian Hamiltonian $\mathcal{H}_{\mathrm{RL}}$ can be realized in this manner, such as the demonstrated $\mathcal{P} \mathcal{T}$-symmetric flat bands [38].

Applying the Fourier transformation to the real-space Hamiltonian, we obtain a six-band Bloch Hamiltonian in the momentum space because of the alternatively introduced gain and loss in the sublattices $B$ and $C$ in the translational direction:

$$
\mathcal{H}_{k}=\left(\begin{array}{cccccc}
0 & G_{1} & G_{1}^{*} & 0 & G_{2}^{*} e^{-i k} & G_{2} e^{-i k} \\
G_{1}^{*} & i \gamma & \delta & G_{2} & 0 & 0 \\
G_{1} & \delta & -i \gamma & G_{2}^{*} & 0 & 0 \\
0 & G_{2}^{*} & G_{2} & 0 & G_{1} & G_{1}^{*} \\
G_{2} e^{i k} & 0 & 0 & G_{1}^{*} & -i \gamma & \delta \\
G_{2}^{*} e^{i k} & 0 & 0 & G_{1} & \delta & i \gamma
\end{array}\right) .
$$

The band energy $E_{k}$ of the Hamiltonian $\mathcal{H}_{k}$ satisfies

$$
\begin{aligned}
0= & E_{k}^{6}+2 E_{k}^{4}\left(\gamma^{2}-\delta^{2}-2 g_{1}^{2}-2 g_{2}^{2}\right) \\
& +E_{k}^{2}\left(\gamma^{2}-\delta^{2}-2 g_{1}^{2}-2 g_{2}^{2}\right)^{2} \\
& +16 g_{1}^{2} g_{2}^{2}\left(\gamma^{2}-\delta^{2}\right) \cos ^{2}(k / 2) .
\end{aligned}
$$

We notice that the band energies are independent of the momentum $k$ for

$$
\gamma^{2}=\delta^{2},
$$

which is the condition for the EP of the non-Hermitian asymmetric rhombic lattice. At the EP, the spectrum is constituted by three coalesced flat bands with energies $-\sqrt{2 g_{1}^{2}+2 g_{2}^{2}}, 0$, and $\sqrt{2 g_{1}^{2}+2 g_{2}^{2}}$.

We first discuss the energy bands and the eigenstates of the non-Hermitian asymmetric rhombic lattice. The gain and loss are alternatively introduced along the translational invariant direction. Unit-cell sites in the lattice expand from three to six, and the band energies are the same as the Hermitian asymmetric rhombic lattice. The difference in the spectrum of the non-Hermitian asymmetry rhombic lattice is that all the energy levels in each flat band are two-state coalesced. We plot the eigenenergies of the real-space Hamiltonian for a 60 -site rhombic lattice as functions of the magnetic flux $\Phi$ as well as the coupling $\delta$ between sublattices $B$ and $C$ in Fig. 3 . Most of the energy levels are doubly degenerate. The $\mathrm{AB}$ cage forms at $\Phi=\pi / 2$, which indicates that the destructive interference occurs when the magnetic flux enclosed in each diamond plaquette is $\pi$ [Figs. 3(a) and 3(b)]. The lower panel
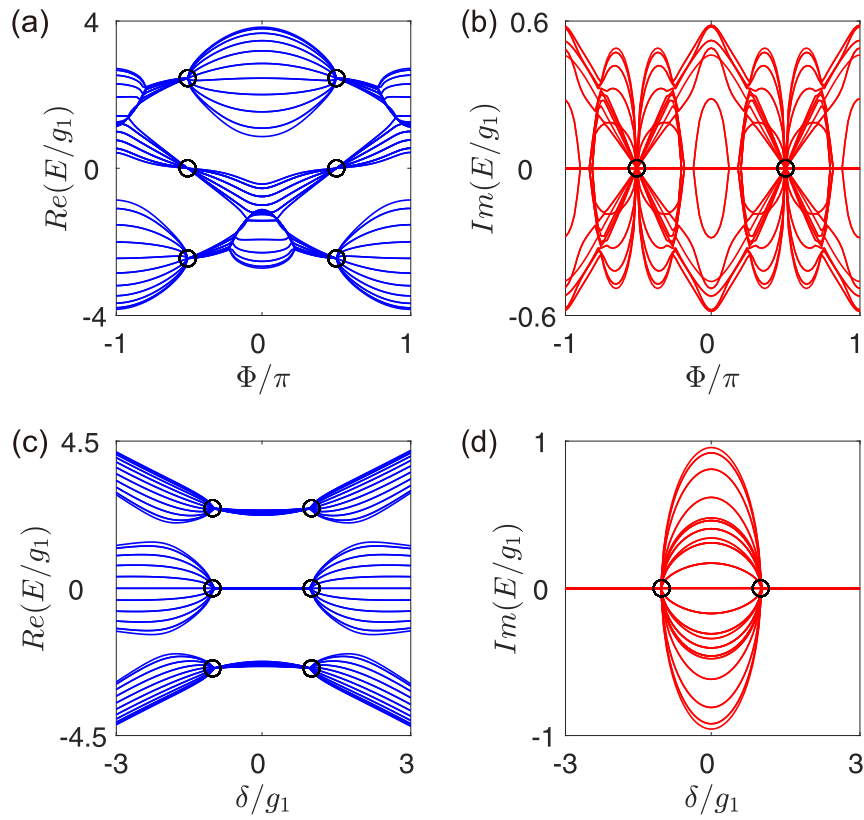

FIG. 3. Spectra of the real-space non-Hermitian asymmetric rhombic lattice as functions of (a), (b) the magnetic flux $\Phi$ and (c), (d) the coupling $\delta$ between sublattices $B$ and $C$. A large amount of energy levels coalesce to three real energies as indicated by the black circles, where the Aharonov-Bohm cage forms. The rhombic lattice has $N=20$ unit cells, being 60 sites in total. The parameters are (a), (b) $g_{1}=1, g_{2}=\sqrt{2}, \delta=\gamma=1$ and (c), (d) $g_{1}=1, g_{2}=\sqrt{2}$, $\gamma=1, \phi=\pi / 2$.

of Fig. 3 shows that the $\mathrm{AB}$ cage appears when the $\mathcal{P} \mathcal{T}$ dimers are at the EPs, that is, $\delta=\gamma$ [Figs. 3(c) and 3(d)].

For the eigenstates, we show that the wave function of the CLS is still unchanged in Figs. 2(c) and 2(d), but the number of the CLSs supported in the non-Hermitian asymmetric rhombic lattice reduces by one-half, which is a consequence of the two-state coalescence at the EP. The discrete Schrödinger equations for the quasi-1D non-Hermitian rhombic lattice are

$$
\begin{aligned}
i \dot{\psi}_{A_{2 j-1}}= & g_{1} e^{i \pi / 4} \psi_{B_{2 j-1}}+g_{1} e^{-i \pi / 4} \psi_{C_{2 j-1}} \\
& +g_{2} e^{-i \pi / 4} \psi_{B_{2 j-2}}+g_{2} e^{i \pi / 4} \psi_{C_{2 j-2}}, \\
i \dot{\psi}_{B_{2 j-1}}= & g_{1} e^{-i \pi / 4} \psi_{A_{2 j-1}}+g_{2} e^{i \pi / 4} \psi_{A_{2 j}} \\
& +\delta \psi_{C_{2 j-1}}+i \gamma \psi_{B_{2 j-1}}, \\
i \dot{\psi}_{C_{2 j-1}}= & g_{1} e^{i \pi / 4} \psi_{A_{2 j-1}}+g_{2} e^{-i \pi / 4} \psi_{A_{2 j}} \\
& +\delta \psi_{B_{2 j-1}}-i \gamma \psi_{C_{2 j-1}}, \\
i \dot{\psi}_{A_{2 j}}= & g_{1} e^{i \pi / 4} \psi_{B_{2 j}}+g_{1} e^{-i \pi / 4} \psi_{C_{2 j}} \\
+ & g_{2} e^{-i \pi / 4} \psi_{B_{2 j-1}}+g_{2} e^{i \pi / 4} \psi_{C_{2 j-1}}, \\
i \dot{\psi}_{B_{2 j}}= & g_{1} e^{-i \pi / 4} \psi_{A_{2 j}}+g_{2} e^{i \pi / 4} \psi_{A_{2 j+1}} \\
& +\delta \psi_{C_{2 j}}-i \gamma \psi_{B_{2 j}}, \\
i \dot{\psi}_{C_{2 j}}= & g_{1} e^{i \pi / 4} \psi_{A_{2 j}}+g_{2} e^{-i \pi / 4} \psi_{A_{2 j+1}} \\
& +\delta \psi_{B_{2 j}}+i \gamma \psi_{C_{2 j}} .
\end{aligned}
$$


The difference between the discrete Schrödinger equations for the Hermitian and non-Hermitian rhombic lattices is reflected from the gain and loss in the sublattices $B$ and $C$ as well as the coupling between them. For the non-Hermitian asymmetric lattice, two additional terms are present in the lower row of the right side of the Schrödinger equations for the sublattices $B$ and $C$. It is straightforward to verify that the steady-state solutions of the Schrödinger equations are the CLSs presented in Figs. 2(c) and 2(d) because the contributions of the additional coupling $\delta$ and the gain (loss) cancel. The CLSs of the non-Hermitian asymmetric lattice are identical to those of the Hermitian asymmetric lattice presented in Figs. 1(c) and 1(d).

Any two sites connected by the coupling $\delta$ are considered as a $\mathcal{P} \mathcal{T}$-symmetric dimer; they are at the EPs when $\delta=\gamma$. To verify the CLSs, we first consider the zero energy flat band. The two additional terms in the Schrödinger equations for $B_{2 j-1}$ cancel, $\delta \psi_{C_{2 j-1}}+i \gamma \psi_{B_{2 j-1}}=$ $\delta\left(i g_{1} / g_{2}\right)+(i \gamma)\left(-g_{1} / g_{2}\right)=0$; the two additional terms in the Schrödinger equations for $C_{2 j-1}$ cancel, $\delta \psi_{B_{2 j-1}}-$ $i \gamma \psi_{C_{2 j-1}}=\delta\left(-g_{1} / g_{2}\right)-(i \gamma)\left(i g_{1} / g_{2}\right)=0$. Similarly, the two additional terms in the Schrödinger equations for $B_{2 j}$ cancel, $\delta \psi_{C_{2 j}}-i \gamma \psi_{B_{2 j}}=\delta(1)-i \gamma(-i)=0$; and the two additional terms in the Schrödinger equations for $C_{2 j}$ cancel, $\delta \psi_{B_{2 j}}+$ $i \gamma \psi_{C_{2 j}}=\delta(-i)+i \gamma(1)=0$. Thus, the CLSs for the Hermitian asymmetric rhombic lattice are also the eigenstates of the non-Hermitian asymmetric rhombic lattice.

In the same manner, the nonzero energy CLSs can be verified to satisfy the Schrödinger equations for the nonHermitian asymmetric rhombic lattice. When considering the time evolution only covering the CLSs, the excitation exhibits constant dynamics. The Hermitian symmetric rhombic lattice can support only one or two flat bands at certain specialized conditions [19]. Although we focus on the situation of the $\mathrm{AB}$ cage with all the three bands being flat, we point out that these interesting situations also exist in the Hermitian asymmetric rhombic lattice; furthermore, the non-Hermitian asymmetric rhombic lattice can similarly support only a single band or only two flat bands. Although the eigenstates of the non-Hermitian asymmetric rhombic lattice are unchanged in comparison with the Hermitian asymmetric rhombic lattice, the CLSs are coalescence states. The dynamics of excitations can exhibit anomalous behaviors in the non-Hermitian asymmetric rhombic lattice if the associated eigenstates of the Jordan block at the EPs are relevant in the time evolution dynamics [50].

\section{LOCALIZATION DYNAMICS IN THE NON-HERMITIAN RHOMBIC LATTICE}

The most significant and intriguing difference for the non-Hermitian asymmetric rhombic lattice is the excitation confinement and the coalesced eigenstates caused by the nonHermitian $\mathcal{P} \mathcal{T}$ dimer at the EP. The influence of eigenstate coalescence is revealed in the localization dynamics. The presented numerical simulation of the dynamics is performed in the rhombic lattice under the periodic boundary condition, while the dynamics performed in the rhombic lattice under the open boundary condition holds the same results. In the numerical simulations, the coupling strengths and the gain (loss) rates are comparable, which are typical parameters
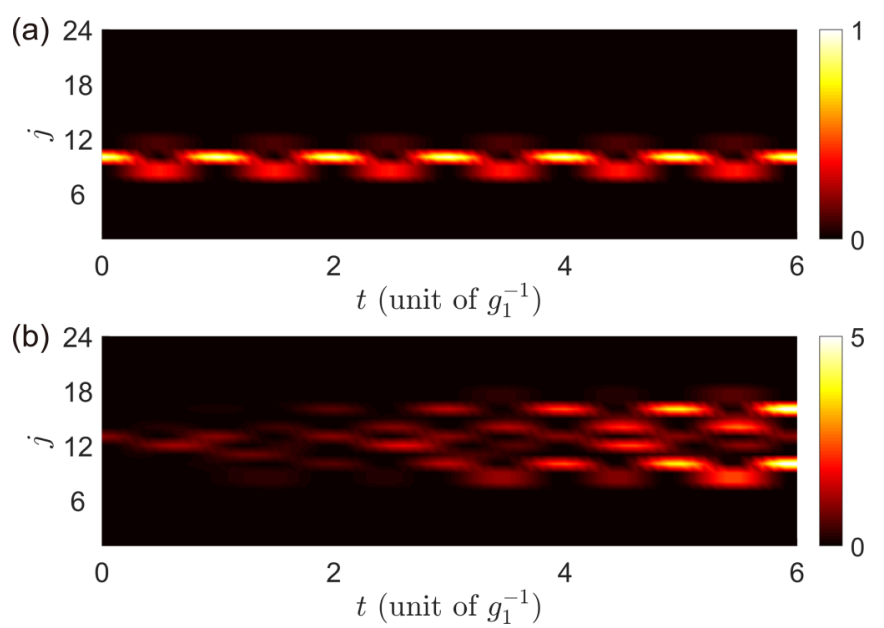

FIG. 4. The localization dynamics with initial excitation of a single site at (a) the site 10 and (b) the site 13. In the lattice of Fig. 2(b), excited sites at time zero are indicated by blue and red arrows for (a) and (b), respectively. In both of the two cases, the parameters are fixed and are $g_{1}=\delta=\gamma=1, g_{2}=2, \Phi=\pi / 2$.

within the reach of the photonic lattice platform of coupled waveguides [21,22,38].

The time evolution processes of different types of initial excitations are simulated in Fig. 4, including two typical localization dynamics performed for the single-site excitations. In Fig. 4(a), the single-site excitation at site 10 is indicated by the blue arrow in Fig. 2(b). The excitation intensity is localized within the region from site 8 to site 12 , indicated by the five-site-cross structure inside the blue rectangle. The evolution period is $2 \pi / \sqrt{2 g_{1}^{2}+2 g_{2}^{2}}$ and the intensity periodically features the brightness and darkness. Notably, the total intensity is a constant 1 although in the non-Hermitian asymmetric lattice. The constant intensity in the time evolution process is a consequence that the initial state only relates to the orthogonal eigenstates of the rhombic lattice. For the single-site excitation in this situation, the dynamics in the non-Hermitian asymmetric rhombic lattice is identical to that in the Hermitian asymmetric rhombic lattice.

To understand this point, it is worth pointing out that the rightward (leftward) unidirectional coupling in Fig. 2(a) relates to the vertical $\mathcal{P} \mathcal{T}$ dimer at the EP with gain (loss) and loss (gain) from the top to the bottom in Fig. 2(b). The single-site excitation in Fig. 2(b) is similar to the single-site excitation at the middle site of the three-site chain cell with inward unidirectional couplings at both sides in Fig. 2(a). The inward unidirectional couplings in Fig. 2(a) ensure that the evolution inside the three-site chain cell is unaffected, being identical to the time evolution of excitation in the isolated three-site chain cell. Consequently, the excitation in the nonHermitian asymmetric rhombic lattice is completely confined in the area inside the blue rectangle and the time evolution dynamics in this situation is identical to that in the Hermitian rhombic lattice.

However, the dynamics exhibited in Fig. 4(b) is distinct from that in Fig. 4(a), where the initial excitation is a singlesite excitation at site 13 indicated by the red arrow in Fig. 2(b). In this situation, the $\mathcal{P} \mathcal{T}$ dimers that close to the red arrow 
induce the excitation tunneling to the two neighbor five-site crosses on the left side and on the right side of the red arrow. The equivalent outward unidirectional couplings in pink in Fig. 2(a) for the $\mathcal{P} \mathcal{T}$ dimers explain the tunneling direction of excitation. The localization area for the single-site excitation at site 13 is indicated by the red rectangle. The confined region of the excitation distribution in Fig. 4(b) is larger in comparison to that in Fig. 4(a). Also, the total intensity for the single-site excitation at site 13 increases polynomially [51], accompanied by the periodic oscillation with a period of $2 \pi / \sqrt{2 g_{1}^{2}+2 g_{2}^{2}}$.

Inspired by the single-site excitation dynamics in the non-Hermitian rhombic lattice, the manipulation of the unitary/nonunitary dynamics and control of the localization area are feasible by changing the distribution of gain and loss. When exciting the central site of the five-site-cross structure as indicated by the red arrow in the schematic of Fig. 5(a), the excitation is fully confined within the five-sitecross structure centered at the excited site. The localization dynamics is performed in Fig. 5(b). The average position, defined as the sum of the product of the lattice positions and the corresponding distribution probabilities, of the excitation is shown in Fig. 5(c). Notably, in the time period of $t=0$ to 5 , the asymmetric rhombic lattice is Hermitian; in the

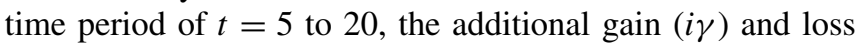
$(-i \gamma)$ are present in site 14 and site 15 , respectively; the two sites are directly coupled through a reciprocal coupling $\delta$. In the whole simulation with sudden change at $t=5$, the separation for results of Hermitian and non-Hermitian models is indicated by the blue dotted lines in Figs. 5(b) and 5(c). The final state in the Hermitian asymmetric lattice at $t=5$ is the initial state of the non-Hermitian asymmetric lattice in the dynamics.

In Fig. 5(b), the dynamics from $t=0$ to 5 is unitary, and the total intensity always keeps unitary. The localization dynamics from $t=5$ to 20 is nonunitary, and the total intensity increases quadratically as a consequence of the eigenstate coalescence at the EP [51]. After $t=5$, another three sites marked from 16 to 18 appear as nonzero probabilities at the right side of the $\mathcal{P} \mathcal{T}$ dimer, and the excitation localization area shifts rightward as indicated by the average position in Fig. 5(c). Also, in the whole time period from $t=0$ to 20 , the oscillation period of the excitation intensity is $2 \pi / \sqrt{2 g_{1}^{2}+2 g_{2}^{2}}$. In contrast to Figs. 5(b) and 5(c), the localization area of the excitation can be tuned leftward if the gain and loss in the rhombic lattice are interchanged as illustrated in Fig. 5(d). And the first subsection in Figs. 5(e) and 5(f) from $t=0$ to 5 is the same as that in Figs. 5(b) and 5(c). The light flow is released to the left side and the excitation intensity increases dramatically. The dynamics is shown in Figs. 5(e) and 5(f); the localization area shifts leftward and the excitation is confined around site 10 due to the presence of the $\mathcal{P} \mathcal{T}$ dimer.

In the above-mentioned two types of localization control, the initial states for the second subsection from $t=5$ to 20 are the same because of the exactly identical dynamics in the time interval from $t=0$ to 5 . However, the dynamics is asymmetric in the second subsection from $t=5$ to 20 , where a $\mathcal{P} \mathcal{T}$ dimer presents on the right side and the left side (a)

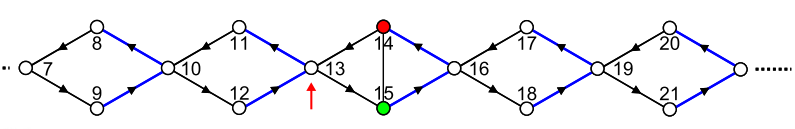

(b)
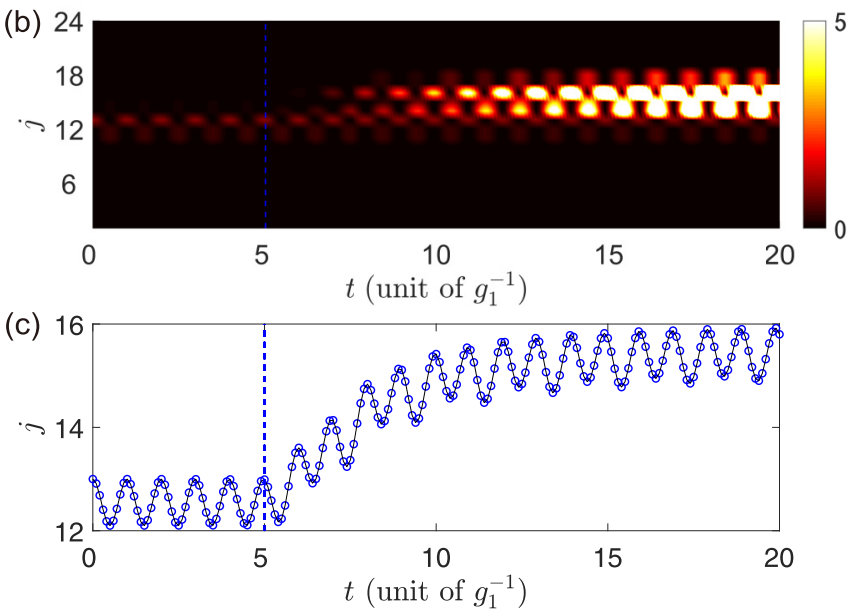

(d)
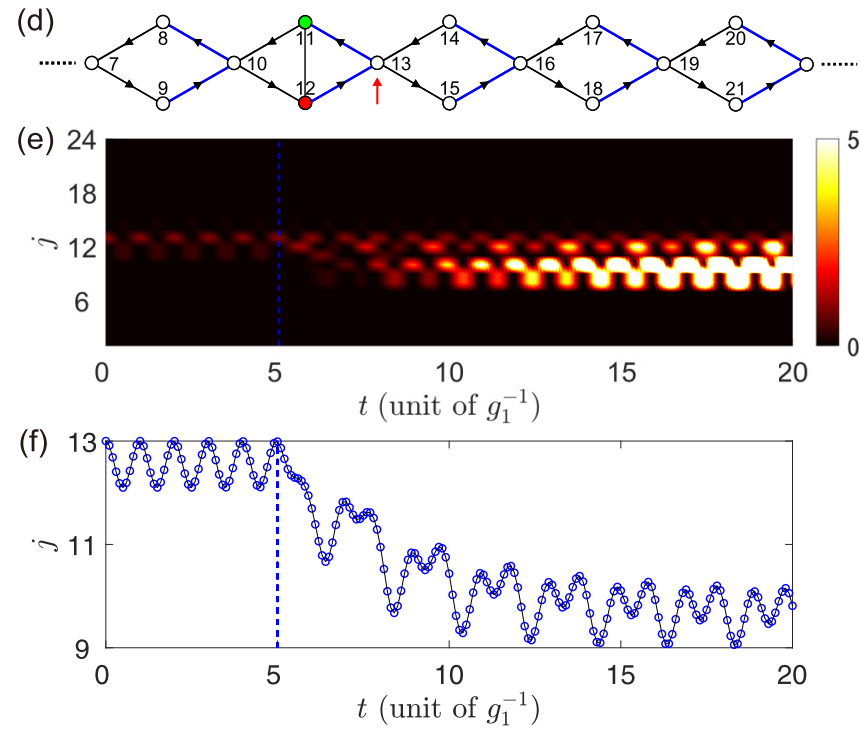

FIG. 5. (a) and (d) are schematics of the asymmetric rhombic lattice. (b) and (e) describe the snapshots of the time evolution dynamics. (c) and (f) represent the average position of excitation. The blue dotted line in (b), (c), (e), and (f) indicates the boundary between the simulation results of the Hermitian and the non-Hermitian lattices. The red arrows in the two schematics point to the excited sites at $t=0$ for the Hermitian models, and the non-Hermitian models shown in (a) and (d) are for the subsections at the right side of the blue lines. The system parameters are $g_{1}=\delta=\gamma=1, g_{2}=2, \Phi=$ $\pi / 2$.

of the excitation, respectively. The asymmetric dynamics is observed through comparing the snapshots and the average position of excitation in Fig. 5. The asymmetric dynamics only emerges when the non-Hermitian rhombic lattice has the asymmetric couplings $g_{1} \neq g_{2}$. For the identical coupling strength $g_{1}=g_{2}$, the dynamics is symmetric.

The asymmetric rhombic lattice of coupled waveguides is advantageous for the light confinement. An arbitrary excitation rather than the specific CLSs only can be confined. The configuration of localization is tunable due to the exact 
control of the lattice parameters in the platform of coupled waveguides. The $\mathcal{P} \mathcal{T}$ dimer in the asymmetric rhombic lattice manipulates the localization area, which is not possible in the Hermitian lattice. This is an application of non-Hermitian metamaterials that provides opportunities for manipulating the light flow. Furthermore, the coupled resonator array is another ideal candidate for the realization of flat bands and $\mathrm{AB}$ caging in the experiment.

\section{CONCLUSION}

We have investigated the Hermitian asymmetric rhombic lattice and elucidated the complete localization confinement from the viewpoint of isolated trimers. We propose the nonHermitian asymmetric rhombic lattice through introducing the $\mathcal{P} \mathcal{T}$ dimers with balanced gain and loss; the orientation of the gain and loss plays a key role in engineering and manipulating the excitation localization of the rhombic lattice. The Aharonov-Bohm cage, fully consisting of flat bands, forms at the EP of the non-Hermitian asymmetric rhombic lattice. And the excitation intensity can be constant or can polynomially increase with periodic oscillation. The confinement mechanism of the non-Hermitian Aharonov-Bohm cage is the isolation induced by the unidirectional coupling. The proposed non-Hermitian asymmetric rhombic lattice can be realized experimentally in the Hermitian rhombic lattices with extra gain and loss or with different losses introduced in the sublattices. Our findings are valuable for the light localization and engineering in the non-Hermitian metamaterials.

\section{ACKNOWLEDGMENT}

We acknowledge the support of National Natural Science Foundation of China (Grant No. 11975128).
[1] C. E. Creffield and G. Platero, Coherent Control of Interacting Particles Using Dynamical and Aharonov-Bohm Phases, Phys. Rev. Lett. 105, 086804 (2010).

[2] N. Masumoto, N. Y. Kim, T. Byrnes, K. Kusudo, A. Löffler, S. Höfling, A. Forchel, and Y. Yamamoto, Exciton-polariton condensates with flat bands in a two-dimensional kagome lattice, New J. Phys. 14, 065002 (2012).

[3] D. Leykam, S. Flach, O. Bahat-Treidel, and A. S. Desyatnikov, Flat band states: Disorder and nonlinearity, Phys. Rev. B 88, 224203 (2013); S. Flach, D. Leykam, J. D. Bodyfelt, P. Matthies, and A. S. Desyatnikov, Detangling flat bands into Fano lattices, Europhys. Lett. 105, 30001 (2014).

[4] J. D. Bodyfelt, D. Leykam, C. Danieli, X. Yu, and S. Flach, Flatbands under Correlated Perturbations, Phys. Rev. Lett. 113, 236403 (2014).

[5] T. Jacqmin, I. Carusotto, I. Sagnes, M. Abbarchi, D. D. Solnyshkov, G. Malpuech, E. Galopin, A. Lemaître, J. Bloch, and A. Amo, Direct Observation of Dirac Cones and a Flatband in a Honeycomb Lattice for Polaritons, Phys. Rev. Lett. 112, 116402 (2014).

[6] R. Khomeriki and S. Flach, Landau-Zener Bloch Oscillations with Perturbed Flat Bands, Phys. Rev. Lett. 116, 245301 (2016).

[7] L. Morales-Inostroza and R. A. Vicencio, Simple method to construct flat-band lattices, Phys. Rev. A 94, 043831 (2016).

[8] F. Baboux, L. Ge, T. Jacqmin, M. Biondi, E. Galopin, A. Lemaître, L. Le Gratiet, I. Sagnes, S. Schmidt, H. E. Türeci, A. Amo, and J. Bloch, Bosonic Condensation and DisorderInduced Localization in a Flat Band, Phys. Rev. Lett. 116, 066402 (2016).

[9] A. Ramachandran, A. Andreanov, and S. Flach, Chiral flat bands: Existence, engineering, and stability, Phys. Rev. B 96, 161104(R) (2017); W. Maimaiti, A. Andreanov, H. C. Park, O. Gendelman, and S. Flach, Compact localized states and flat-band generators in one dimension, ibid. 95, 115135 (2017).

[10] M. Tovmasyan, S. Peotta, L. Liang, P. Törmä, and S. D. Huber, Preformed pairs in flat Bloch bands, Phys. Rev. B 98, 134513 (2018).
[11] D. Leykam, A. Andreanov, and S. Flach, Artificial flat band systems: From lattice models to experiments, Adv. Phys. X 3, 1473052 (2018).

[12] D. Guzmán-Silva, C. Mejía-Cortés, M. A. Bandres, M. C. Rechtsman, S. Weimann, S. Nolte, M. Segev, A. Szameit, and R. A. Vicencio, Experimental observation of bulk and edge transport in photonic Lieb lattices, New J. Phys. 16, 063061 (2014).

[13] S. Mukherjee and R. R. Thomson, Observation of localized flat-band modes in a quasi-one-dimensional photonic rhombic lattice, Opt. Lett. 40, 5443 (2015).

[14] R. A. Vicencio, C. Cantillano, L. Morales-Inostroza, B. Real, C. Mejía-Cortés, S. Weimann, A. Szameit, and M. I. Molina, Observation of Localized States in Lieb Photonic Lattices, Phys. Rev. Lett. 114, 245503 (2015).

[15] S. Mukherjee, A. Spracklen, D. Choudhury, N. Goldman, P. Öhberg, E. Andersson, and R. R. Thomson, Observation of a Localized Flat-Band State in a Photonic Lieb Lattice, Phys. Rev. Lett. 114, 245504 (2015).

[16] S. Mukherjee and R. R. Thomson, Observation of robust flatband localization in driven photonic rhombic lattices, Opt. Lett. 42, 2243 (2017).

[17] L. Ge, Anomalous minimum and scaling behavior of localization length near an isolated flat band, Ann. Phys. (Berlin) 529, 1600182 (2017).

[18] J. Vidal, B. Douçot, R. Mosseri, and P. Butaud, Interaction Induced Delocalization for Two Particles in a Periodic Potential, Phys. Rev. Lett. 85, 3906 (2000).

[19] Z. Gulácsi, A. Kampf, and D. Vollhardt, Exact Many-Electron Ground States on the Diamond Hubbard Chain, Phys. Rev. Lett. 99, 026404 (2007).

[20] S. Longhi, Aharonov-Bohm photonic cages in waveguide and coupled resonator lattices by synthetic magnetic fields, Opt. Lett. 39, 5892 (2014).

[21] S. Mukherjee, M. Di Liberto, P. Öhberg, R. R. Thomson, and N. Goldman, Experimental Observation of Aharonov-Bohm Cages in Photonic Lattices, Phys. Rev. Lett. 121, 075502 (2018). 
[22] M. Kremer, I. Petrides, E. Meyer, M. Heinrich, O. Zilberberg, and A. Szameit, A square-root topological insulator with non-quantized indices realized with photonic Aharonov-Bohm cages, Nat. Commun. 11, 907 (2020).

[23] G. Gligorić, P. P. Beličev, D. Leykam, and A. Maluckov, Nonlinear symmetry breaking of Aharonov-Bohm cages, Phys. Rev. A 99, 013826 (2019).

[24] M. Di Liberto, S. Mukherjee, and N. Goldman, Nonlinear dynamics of Aharonov-Bohm cages, Phys. Rev. A 100, 043829 (2019).

[25] J. Vidal, R. Mosseri, and B. Douçot, Aharonov-Bohm Cages in Two-Dimensional Structures, Phys. Rev. Lett. 81, 5888 (1998); J. Vidal, P. Butaud, B. Douçot, and R. Mosseri, Disorder and interactions in Aharonov-Bohm cages, Phys. Rev. B 64, 155306 (2001).

[26] S. Longhi, Parity-time symmetry meets photonics: A new twist in non-Hermitian optics, Europhys. Lett. 120, 64001 (2017).

[27] L. Feng, R. El-Ganainy, and L. Ge, Non-Hermitian photonics based on parity-time symmetry, Nat. Photon. 11, 752 (2017).

[28] R. El-Ganainy, K. G. Makris, M. Khajavikhan, Z. H. Musslimani, S. Rotter, and D. N. Christodoulides, NonHermitian physics and $\mathcal{P} \mathcal{T}$ symmetry, Nat. Phys. 14, 11 (2018).

[29] S. K. Gupta, Y. Zou, X.-Y. Zhu, M.-H. Lu, L.-J. Zhang, X.-P. Liu, and Y.-F. Chen, Parity-time symmetry in non-Hermitian complex optical media, Adv. Mater. 32, 1903639 (2019).

[30] D. Christodoulides and J. Yang, Parity-Time Symmetry and Its Applications (Springer, Singapore, 2018).

[31] S. K. Özdemir, S. Rotter, F. Nori, and L. Yang, Parity-time symmetry and exceptional points in photonics, Nat. Mater. 18, 783 (2019).

[32] M.-A. Miri and A. Alù, Exceptional points in optics and photonics, Science 363, eaar7709 (2019).

[33] M. I. Molina, Flat bands and $\mathcal{P} \mathcal{T}$ symmetry in quasi-onedimensional lattices, Phys. Rev. A 92, 063813 (2015).

[34] L. Ge, Parity-time symmetry in a flat-band system, Phys. Rev. A 92, 052103 (2015).

[35] D. Leykam, S. Flach, and Y. D. Chong, Flat bands in lattices with non-Hermitian coupling, Phys. Rev. B 96, 064305 (2017).

[36] B. Qi, L. Zhang, and L. Ge, Defect States Emerging from a Non-Hermitian Flatband of Photonic Zero Modes, Phys. Rev. Lett. 120, 093901 (2018).

[37] H. Ramezani, Non-Hermiticity-induced flat band, Phys. Rev. A 96, 011802(R) (2017).
[38] T. Biesenthal, M. Kremer, M. Heinrich, and A. Szameit, Experimental Realization of $\mathcal{P} \mathcal{T}$-Symmetric Flat Bands, Phys. Rev. Lett. 123, 183601 (2019).

[39] L. Jin, Flat band induced by the interplay of synthetic magnetic flux and non-Hermiticity, Phys. Rev. A 99, 033810 (2019).

[40] S. M. Zhang and L. Jin, Flat band in two-dimensional nonHermitian optical lattices, Phys. Rev. A 100, 043808 (2019).

[41] M. Hafezi, S. Mittal, J. Fan, A. Migdall, and J. M. Taylor, Imaging topological edge states in silicon photonics, Nat. Photon. $\mathbf{7}$ 1001 (2013).

[42] M. Hafezi, Synthetic gauge fields with photons, Int. J. Mod. Phys. B 28, 1441002 (2014); Measuring Topological Invariants in Photonic Systems, Phys. Rev. Lett. 112, 210405 (2014).

[43] P. St-Jean, V. Goblot, E. Galopin, A. Lemaître, T. Ozawa, L. Le Gratiet, I. Sagnes, J. Bloch, and A. Amo, Lasing in topological edge states of a one-dimensional lattice, Nat. Photon. 11, 651 (2017).

[44] M. Parto, S. Wittek, H. Hodaei, G. Harari, M. A. Bandres, J. Ren, M. C. Rechtsman, M. Segev, D. N. Christodoulides, and M. Khajavikhan, Edge-mode Lasing in 1D Topological Active Arrays, Phys. Rev. Lett. 120, 113901 (2018).

[45] H. Zhao, P. Miao, M. H. Teimourpour, S. Malzard, R. ElGanainy, H. Schomerus, and L. Feng, Topological hybrid silicon microlasers, Nat. Commun. 9, 981 (2018).

[46] C. Poli, M. Bellec, U. Kuhl, F. Mortessagne, and H. Schomerus, Selective enhancement of topologically induced interface states in a dielectric resonator chain, Nat. Commun. 6, 6710 (2015).

[47] S. Weimann, M. Kremer, Y. Plotnik, Y. Lumer, S. Nolte, K. G. Makris, M. Segev, M. C. Rechtsman, and A. Szameit, Topologically protected bound states in photonic parity-time-symmetric crystals, Nat. Mater. 16, 433 (2017).

[48] M. Pan, H. Zhao, P. Miao, S. Longhi, and L. Feng, Photonic zero mode in a non-Hermitian photonic lattice, Nat. Commun. 9, 1308 (2018).

[49] A. Cerjan, S. Huang, M. Wang, K. P. Chen, Y. Chong, and M. C. Rechtsman, Experimental realization of a Weyl exceptional ring, Nat. Photon. 13, 623 (2019).

[50] P. Wang, L. Jin, G. Zhang, and Z. Song, Wave emission and absorption at spectral singularities, Phys. Rev. A 94, 053834 (2016).

[51] L. Ge, Non-Hermitian lattices with a flat band and polynomial power increase, Photon. Res. 6, A10 (2018). 